\title{
The Kingdom of Saudi Arabia Vehicle License Plate Recognition using Learning Vector Quantization Artificial Neural Network
}

\author{
Yusuf Perwej, Ph.D \\ Ph.D, M.Tech (Computer \\ Science \& Engg.) \\ Assistant Professor \\ Department of Computer \\ Science \& Engg \\ Al Baha University, Al Baha, \\ Kingdom of Saudi Arabia
}

(KSA)

\author{
Nikhat Akhtar \\ M.Tech, B.Tech (Computer \\ Science \& Engg.) \\ Assistant Professor \\ Department of Computer \\ Science \& Engg. \\ Integral University, Lucknow, \\ India
}

\author{
Firoj Parwej \\ Assistant Professor \\ Department of CS \& IS \\ Jazan University, Jazan \\ Kingdom of Saudi Arabia
}

(KSA)

\begin{abstract}
In the today scenario technological intelligence is a higher demand after commodity even in traffic-based systems. These intelligent systems do not only help in traffic monitoring but also in commuter safety, law enforcement and commercial applications. The proposed Saudi Arabia Vehicle License plate recognition system splits into three major parts, firstly extraction of a license plate region secondly segmentation of the plate characters and lastly recognition of each character. This act is quite challenging due to the multiformity of plate formats and the nonuniform outdoor illumination conditions during image collection. In this paper recognition of the license plates is achieved by the implementation of the Learning Vector Quantization artificial neural network. Their results are based upon their completeness in the Saudi Arabia Vehicle License plate character recognition and theirs have obtained encouraging results from proposed technique.
\end{abstract}

\section{Keywords}

Arabic Character Segmentation, Learning Vector Quantization Neural Network, Fan-Beam Feature Extraction, Vehicle License Plate, Extraction.

\section{INTRODUCTION}

The License Plate Recognition system is a very significant part of the Intelligent Transportation System [1] which is very significant for the development in the transport infrastructure of the world [2]. In the developing countries such as a Kingdom of Saudi Arabia. The most common solutions to license plate localization in digital images are through the implementation of edge extraction in this edge viewpoint is normally simple and fast, but it is sentient towards noise [3], histogram analysis in this basic histogram approach is not competent of dealing with images with cogitable amount of noise and tilted license plates [4], and morphological operators in this the localization of license plates via morphological based approaches is not high strung to noise but is very slow in execution [5].

Furthermore though there are many prescripts proposed for License Plate Recognition system. But, there are not single prescripts can provide good performance in all the applications in different complicated background such as the plate is small, Incertitude of edges, low or high illuminated images, different types of plate, size, dim lighting, character fonts, syntax, weather and environment, spacing etc. Therefore, most of the previous prescripts could not apply for all the countries in the real world, all the environments, all types of the Kingdom of Saudi Arabia Vehicle License Plate. The Kingdom of Saudi Arabia Vehicle license plate localization and recognition system today will be required to operate robustly in environments with intricate backgrounds and light intensity variations. To deal with such problems, researchers have proposed in this paper uses Artificial Neural Network techniques [6] for character recognition, using Learning Vector Quantization Neural Network [7]. Their results are compared based upon their completeness in the character recognition. The efficiency of the System can be further improved by increasing the number of fonts for training Learning Vector Quantization Artificial Neural Networks.

\section{THE SAUDI ARABIA VEHICLE PLATE EXTRACTION}

The Arabic License plate extraction is the most vital phase in the Saudi Arabia vehicle license plate recognition system. The several models have been proposed for extraction and recognition aside, but very few of them propose both an extraction as well as recognition. Several of the researchers shown in research papers rely on the fact that the number plate edges are appropriately detected and that the ratio of the width to height of the number plate is a constant [8]. Several of the researchers shown in research papers try to enforce morphological operations to highlight the probable regions of the number plate and afterward filter out all but one. Several other methods such as color edge detection and fuzzy systems, edge statistics and morphological operations, and weight based density maps. In this paper proposed prescript is designed for the Kingdom of Saudi Arabia Vehicle License Plate extraction. Input to the system is an image which contains the license plate, procured from about six meters away by a digital camera on the front or rear of the vehicle. The Kingdom of Saudi Arabia vehicle License Plate method based on Tophat-Bothat transshipment and Line Scanning. The prescript includes the following major stages [9], which are RGB to gray-scale sea-change in other words this image is then converted to a gray scale image the next step is image pre-processing for which Tophat and Bothat technique is used, vertical edge detection and image binarization, analysis and dilation, vertical projection and thresholding, extracting 
the actual position of the Arabic license plate, filtration and image enlargement, binarization and smoothing process [10]. These techniques are mended the overall contrast of the image as shown in the figure 1 .

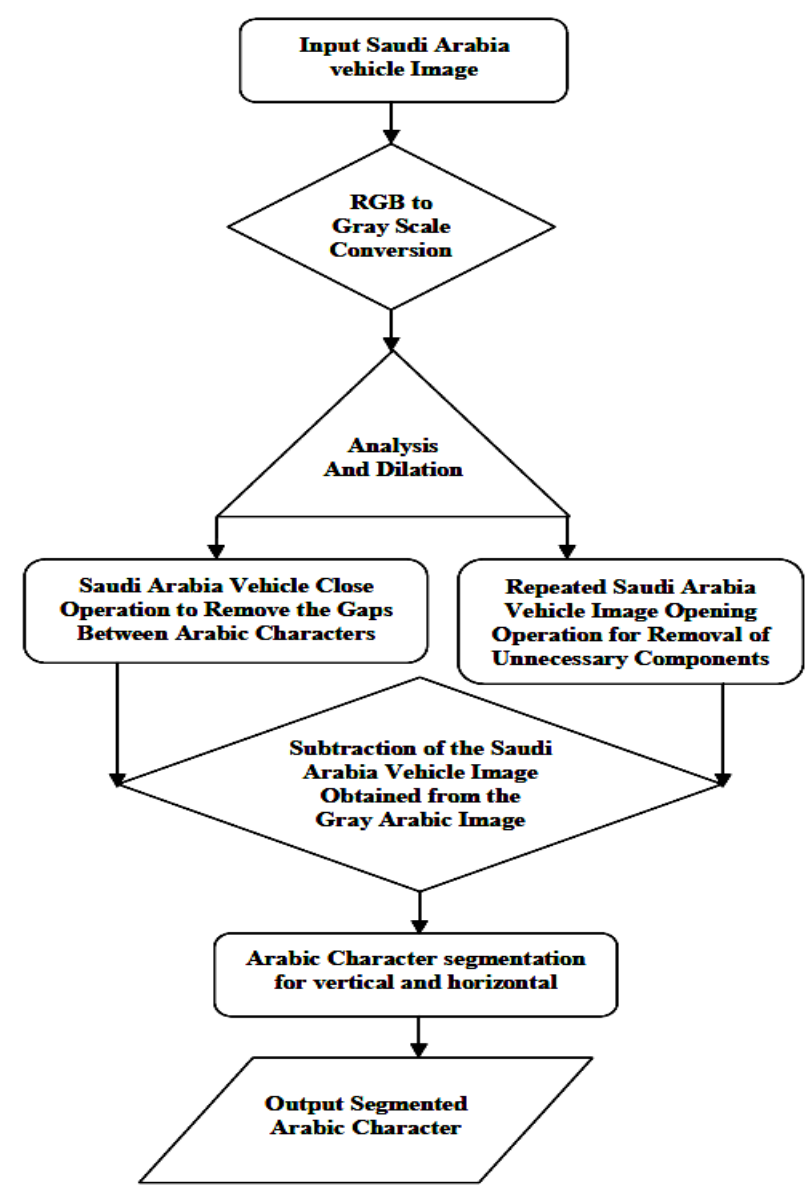

Figure 1. The Saudi Arabia vehicle Plate Extraction

\section{THE SAUDI ARABIA VEHICLE CHARACTER SEGMENTATION}

The vehicle license plate character segmentation is the procedure of extracting the characters from the Arabic license plate image.

The character segmentation is an essential stage because the extent one can reach in partition of words lines or characters directly make an impression the recognition rate of the Saudi Arabia vehicle license plate [11]. The Saudi Arabia vehicle license plate scanning done from right to left of the license plate. In this paper, we proposed first row \& second row of Saudi Arabia vehicle license plate character segmentation, which harmonize pre-processing to disassemble noises, normalized and then segment Arabic characters \& Arabic numbers based.

The quantization of gray image, a black \& white image laid down by an adaptive threshold, Then, the image is resized in parameter 100x150 pixels for first row Saudi Arabia vehicle license plate and 150x100 pixels for second row Saudi Arabia vehicle license plate, [12] shown in the figure 2. After preprocessing work, we need to split second rows in the second row Saudi Arabia vehicle license plate counterpart by computing and a partition in the vertical direction, and then Arabic characters segmentation work is ended.

The segmentation the Arabic characters in digital images in pursuance of to the variable Arabic digit width and regress a matrix containing the two bounds of each Arabic digit. The function lay in the outcome only segment whose rectangular areas are more than min area Arabic digit.

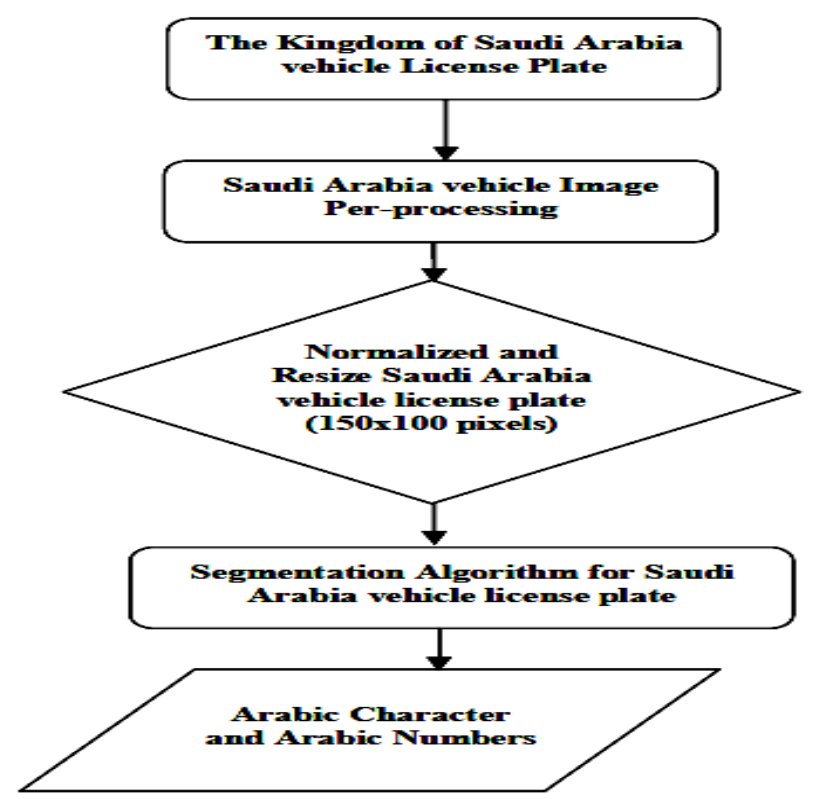

Figure 2. The Saudi Arabia vehicle Character Segmentation

\section{THE SAUDI ARABIA VEHICLE FEATURE EXTRACTION}

The transforming the input data into the set of features is called feature extraction. If the features extracted are discreetly handpicked it is expected that the features set will extract the episodic information from the input data in order to execute the desired task using this diminished representation instead of the full size input [13]. Feature extraction is a usual term for prescript of constructing combinations of the variables to get around these problems while still depicts the data with substantial precision.

There are numerous causes why feature extraction is a vital problem in predictive modeling and modern data analysis. In first cause dimension lack if the problems with a large number of variables, almost all prediction models suffers from the curse of dimensionality, some more harshly than others. Feature extraction can act as an impressive dimension deficiency agent. We can comprehend the curse of dimensionality in very intuitive terms when a person is given too many variables to consider, most of which are inconsequential or simply non-informative; it is naturally much harder to make a better verdict. It is therefore desirable to select a much smaller number of episodic and vital features.

The high dimensional problems also pretence problems for computation. Infrequently two variables might be uniformly informative, but are highly correlated with each other this often causes unhealthy behavior in numerical computation. Therefore feature extraction is a vital computational technique. In another reason automatic exploratory data 
analysis. If numerous classical applications, informative features are often selected a priori by field experts, i.e. explorer pick out what they reckon are the vital variables in making a model. The more often in modern data-mining applications however, there is an accrescent demand for fully automated "black-box" type of prognostication models that are competent of identifying the vital features on their own [14]. The necessity for such automated systems arises for two reasons. Firstly, on the one hand, there are the economic necessities to process large amounts of data in a short amount of time with meager manual supervision. Secondly, on the other hand, it is mostly the case that the problem and the data are so novel that there are simply no field experts who understand the data well enough to be able to pick out the vital variables former to the analysis. The beneath such situation automatic exploratory data analysis becomes the key. Instead of keeping faith on pre-conceived consideration, there is a necessity to let the data speak for itself.

Another motive data visualization. If the application of feature extraction that shares the flavor of exploratory data analysis is data visualization. The human eye has an amazing ability in recognizing systematic patterns in the data. At the same time, we are ordinarily unable to make good sense of data if it is more than three dimensional. To maximize the use of the ultra improved human faculty in visual identification, we oftentimes wish to identify two or three of the most informative features in the data so that we can plot the data in a diminished space. The prescript of feature extraction is described in below [15]. $F(x, y)$ is the severity of the pixel ( $x$, $y),(x c, y c)$ is the position of the present tracking point of the line tracking in the image, $\mathrm{Rf}$ is the set of pixels inside the finger's outline, and $\operatorname{Tr}$ is the locus space. Assume that the pixel in the lower left in the image to be $(0,0)$, the positive direction of the $\mathrm{x}$-axis to be rightward in the image, and the positive direction of the y-axis to be upward inside the image, and $\operatorname{Tr}(\mathrm{x}, \mathrm{y})$ to be initialized to 0 .

Step 1: In this step determination of the beginning point for line tracking and the moving-direction attribute

Step 2: In this step ascertains of the direction of the dark line and agitation of the tracking point

Step 3: In this step updating the number of time points in the locus space have been tracked

Step 4: In this step encore execution of step 1 to step 3 (N times)

Step 5: In this step acquisition of the finger-vein pattern from the locus Space

In this paper, we are proposing feature extraction techniques are tried for training and simulating Learning Vector Quantization Artificial Neural Network using Fan-beam Transform. Feature Extraction is performed on each segmented Arabic character [16].

The Fan beam projection is a variation of radon transform. The fan-beam function computes projections of an image matrix along specified directions except that the projections are taken in a dissimilar way from that of radon transform.

Features were computed using fan-beam geometry. For Fanbeam, 55 diverging beams are taken. The first step is to lay down the distance $\mathrm{D}$ from the fan-beam source to the center of rotation in figure 3 . D must be big sufficient to ensure that the fan-beam source is outside of the image rotation at all angles. $\mathrm{D}$ is taken a few pixels larger than half the diagonal image distance, where the diagonal image distance is, $d=\sqrt{ } \mathrm{i} 2-\mathrm{j} 2$ where $i$ and $j$ are rows and columns of the image serially. Fanbeam takes projections at different angles by rotating the source around the center pixel at $\theta$ degree intervals [17]. These projection data are presumed as feature vectors.

It can be seen from the accumulator data of Fan beam that after 180 degrees the signals encore itself in the head over heels direction. This is because projections taken from 0 to 180 degrees are exactly equal to the projections taken from 181 to 360 degrees [18]. The average value of the procured projection data is computed in order to build the feature vector. For Fan-beam the average of the projections of one direction which is the average of 55 parallel projections was computed. The size of feature vector for one numeral is $1 \times 360$.

The column of fan beam data of the image is 540 sensor samples. It is a common observation in Learning Vector Quantization Artificial neural network system that, if the network is trained with more number of features the simulation accuracy increases. The numbers of features are more in fan-beam feature extraction; Character Recognition accuracy is superior for fan-beam feature extraction than other feature extraction technique.

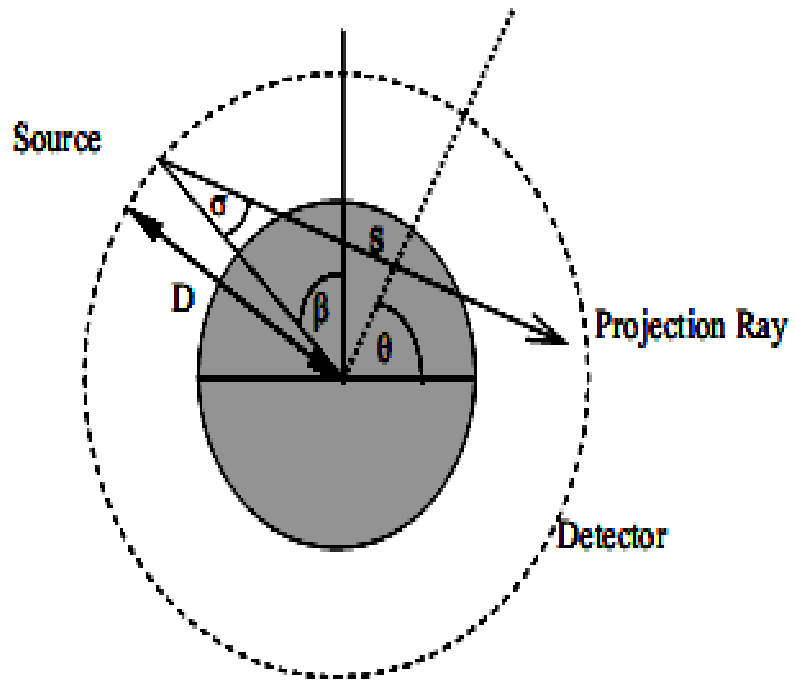

Figure 3. The Fan-beam Geometry

\section{THE PROPOSED MODEL FOR SAUDI ARABIA VEHICLE LICENCE PLATE CHARACTER RECOGNITION}

In this section, a new algorithm for Arabic character recognition is developed which is based on learning vector quantization artificial neural network. It is expected that this procedure works well even in a Kingdom of Saudi Arabia vehicle license plate low resolution images. In the literature we can find several vehicle license plate character recognition methods. In firstly some vehicle license plate matching viewpoint use cross correlation to all possible template shifts over the image while others use hausdorff distance or mean squared error as ours. In secondly some methods used for number plate recognition were optical character recognition and formula based recognition. As learning vector quantization artificial neural network is an intelligent engine, it ensures greater accuracy rate along with the better recognition speed of Kingdom of Saudi Arabia vehicle license plate.

Artificial Neural Network (ANN) is an interdisciplinary study of biology and computer science [19], which has been widely 
used in signal processing, pattern recognition, computer vision, intelligent control, nonlinear optimization, and so on. Learning vector quantization artificial neural network algorithm [20] is one of the most influential methods of Artificial Neural Network. . Learning Vector Quantization (LVQ) has been introduced by T. Kohonen as a simple [21], a universal and efficient classification algorithm and has since found many applications and extensions.

After extracting features from the Kingdom of Saudi Arabia vehicle license plate Arabic character images, it's time to construct the network. In this paper, the number of input nodes is the dimensions of image's eigenvector, and the number of output nodes is the binary encoding with the number of Saudi Arabia vehicle license plate character (Arabic character 28, Arabic numeral character 10).

A Learning Vector Quantization network has a first competitive layer and a second linear layer. The competitive layer learns to classify input vectors in much the same way as the competitive layers. The linear layer transforms the competitive layer's classes into target classifications defined by the user. We refer to the classes learned by the competitive layer as subclasses and the classes of the linear layer as target classes [22].

The core of Learning Vector Quantization neural network is based on the nearest-neighbor method of calculating the Euclidean distance. The distances between each input vector and competitive layer neural nodes can be calculated [23], and the output node which is of minimum gap is designated as a winning node.

$\mathrm{d}(\mathrm{X}, \mathrm{Wc})=\min \{\mathrm{d}(\mathrm{X}, \mathrm{Wi})\},(\mathrm{i}=1,2, \cdots, \mathrm{n})$, where $\mathrm{X}$ is the input vector, Wi is the reference vector,

$\mathrm{d}(\mathrm{X}, \mathrm{Wi})$ is the gap between $\mathrm{X}$ and $\mathrm{Wi}$, and $\mathrm{Wc}$ is the winner subclass. The following equations define the basic Learning Vector Quantization algorithm process

When $\mathrm{i}=\mathrm{c}$, if $\mathrm{X}$ and $\mathrm{Wc}$ belong to the same class

$\mathrm{Wc}(\mathrm{t}+1)=\mathrm{Wc}(\mathrm{t})+\alpha(\mathrm{t})[\mathrm{X}(\mathrm{t})-\mathrm{Wc}(\mathrm{t})]$

if $\mathrm{X}$ and $\mathrm{Wc}$ belong to the different classes

$\mathrm{Wc}(\mathrm{t}+1)=\mathrm{Wc}(\mathrm{t})-\alpha(\mathrm{t})[\mathrm{X}(\mathrm{t})-\mathrm{Wc}(\mathrm{t})]$,

When i $6=c$

$\mathrm{Wi}(\mathrm{t}+1)=\mathrm{Wi}(\mathrm{t})$,

Where $0<\alpha(\mathrm{t})<1$, and learning rate $\alpha(\mathrm{t})$ is ordinarily made to decrease monotonicity with time. It plays a very vital role in network convergence.

By the iterative learning, the input vector $\mathrm{X}$ will be assigned to the class which the reference vector $\mathrm{W}$ belongs to. The class of each input vector can be procured through the competitive learning process.

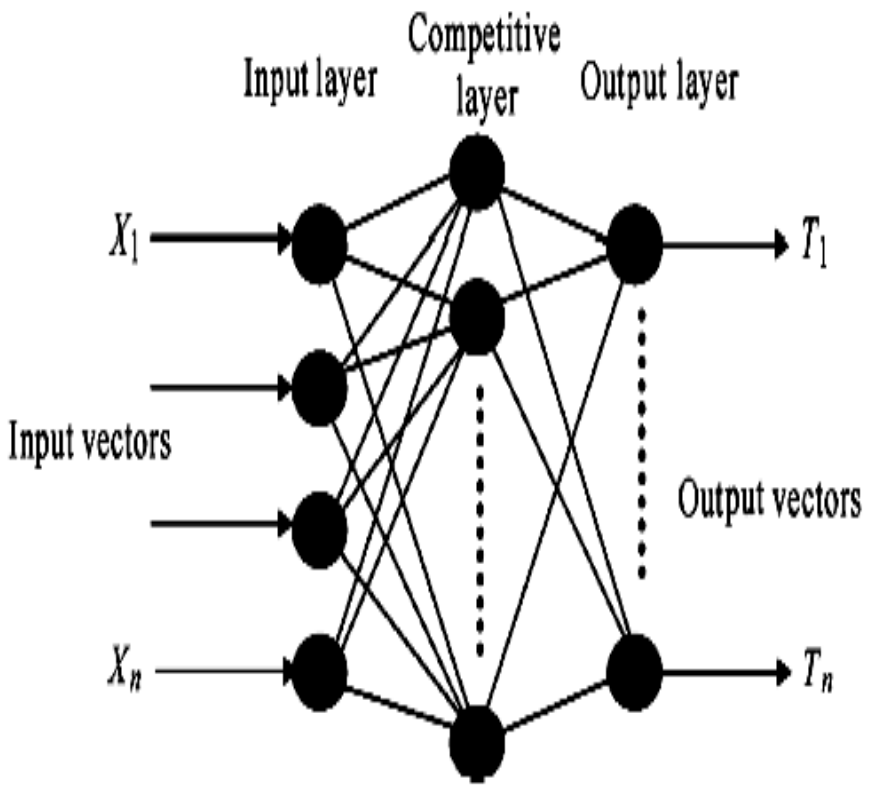

Figure 4. Schematic depiction of LVQ neural network.

Both the competitive and linear layers have one neuron per (sub or target) class. Thus, the competitive layer can learn up to $S^{1}$ subclasses. These, in turn, are combined with the linear layer to form $S^{2}$ target classes. ( $S^{1}$ is always larger than the S2.)

For example, assume that the neurons 1, 2, and 3 in the competitive layer all learn subclasses of the input space that belongs to the linear layer target class no. 2 . Then competitive neurons 1,2 , and 3 , will have $\mathrm{LW}^{2,1}$ weights of 1.0 to neuron $\mathrm{n}^{2}$ in the linear layer, and weights of 0 to all other linear neurons. Thus, the linear neuron output a 1 if any of the three competitive neurons $(1,2$, and 3$)$ win the competition and output a 1 . This is how the subclasses of the competitive layer are combined into target classes in the linear layer.

In short, a 1 in the $i^{\text {th }}$ row of $a^{1}$ (the rest to the elements of $\mathrm{a}^{1}$ will be zero) effectively select the $i^{\text {th }}$ column of $L^{2,1}$ as the network output. Each such column contains a single 1, corresponding to a conspicuous class. Accordingly, subclass $1 \mathrm{~s}$ from layer 1 get put into different classes, by the $\mathrm{LW}^{2,1} \mathrm{a}^{1}$ multiplication in layer 2 .

We know ahead of time what fraction of the layer 1 neurons should be classified into the various class outputs of layer 2, we can specify the elements of $\mathrm{LW}^{2,1}$ at the start. We have to go through a training procedure to get the first layer to produce the correct subclass output for each vector of the training set. 


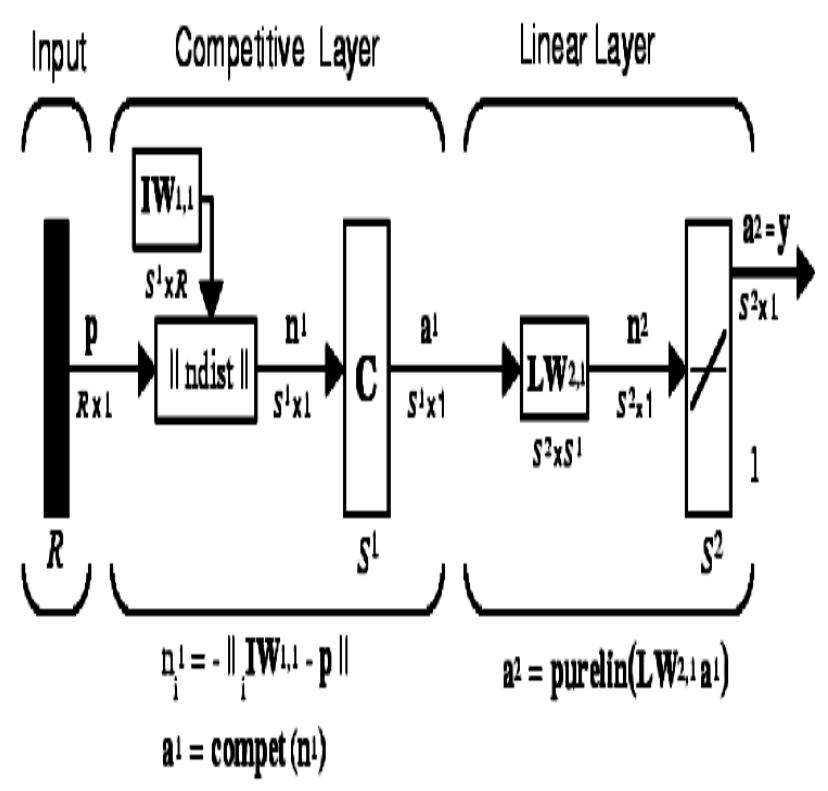

Figure 5. The LVQ network architecture.

After extracting features from the Kingdom of Saudi Arabia vehicle license plate Arabic character images, it's time to construct the network. In this paper, the number of input nodes is the dimensions of image's eigenvector, and the number of output nodes is the binary encoding with the number of Saudi Arabia vehicle license plate character (Arabic character 28, Arabic numeral character 10). The learning vector quantization artificial neural network combines competitive learning with supervision [24]. Target vector is in log sigmoid form (identity matrix). The learning rate is 0.01 for training the network.

\section{EXPRIMENTAL RESULTS OF THE KINDOM OF SAUDI ARABIA VEHICLE LICENCE PLATE RECOGNITION}

We tested Kingdom of Saudi Arabia Vehicle License Plate images which received from the actual system, these vehicle images are very different background, such as Ignis, license angles, size and type, Sandstorm, and dimensions from camera to vehicles, colors, light conditions in the Kingdom of Saudi Arabia environment, these vehicle images are RGB true-color image. In the license plate location module, most of limitations in the previous methods (restricted in case: uncertainty of edges, various types, low or high ignis images, colors, sun light $\&$ night light) were solved by our proposed method by using learning vector quantization artificial neural network.

We implemented experiment with PC Intel(R) Core(TM)2 Duo CPU T7250 @2.40GHz, RAM 4.00 GB, Windows 7 32bit Operating System and MATLAB Version 7.8.0.347 (R2009a).

we have used, subtract operation on the grayscale image to receive a better image with new severity values that satisfied for the Arabic image binarization, this is the reason why our proposed method is very efficient for complex Arabic background images, night \& day images and different Ignis.

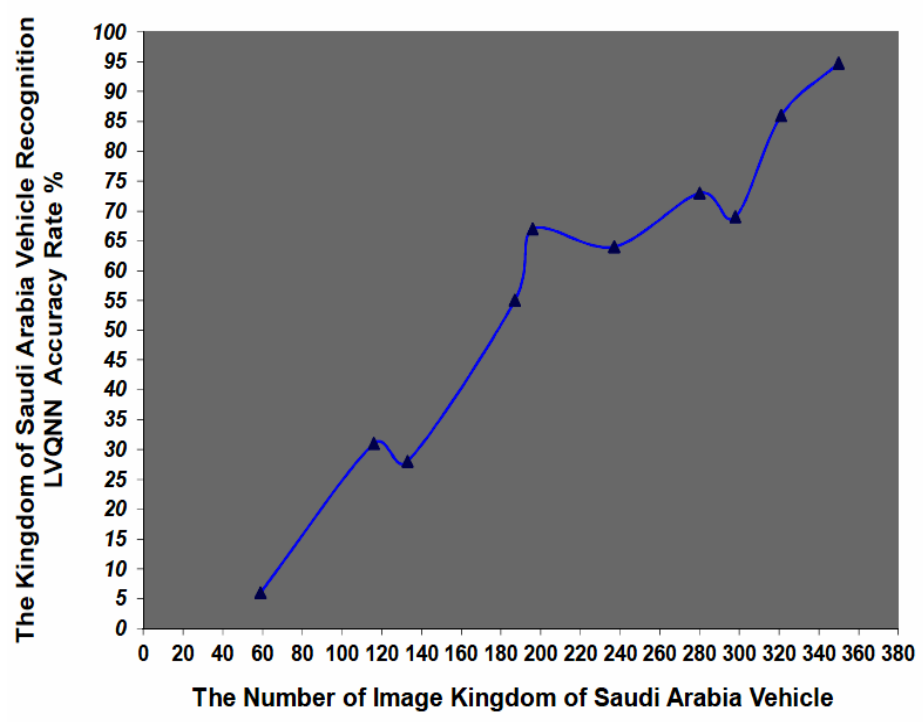

Figure 6. The Saudi Arabia Vehicle License Plate Segmentation

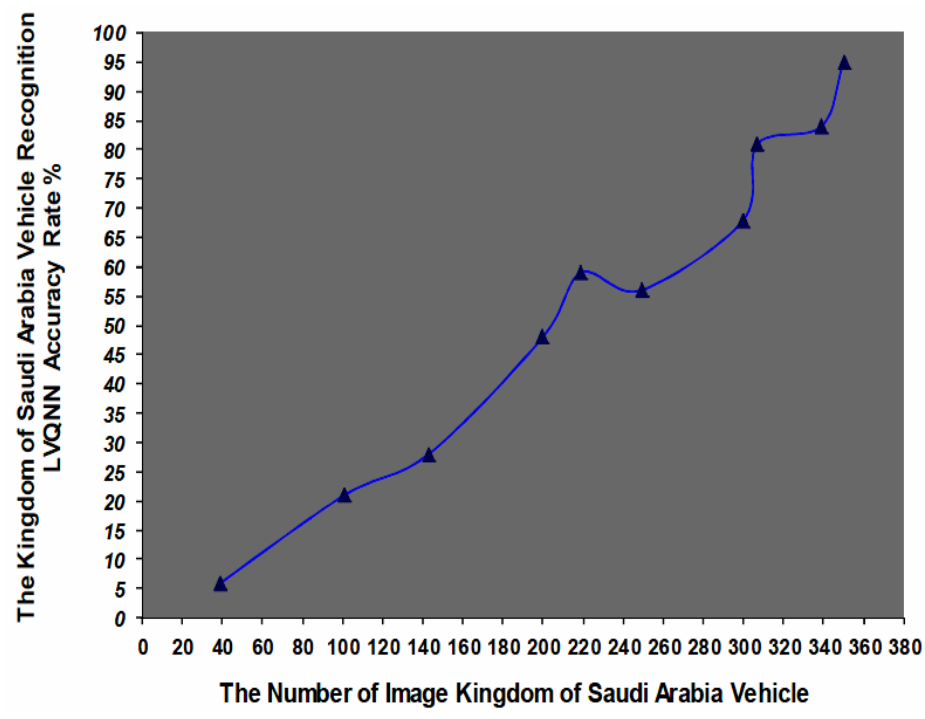

Figure 7. The Saudi Arabia Vehicle License Plate Recognition

we have selected the best values in the fixing the Saudi Arabia vehicle license plate region step, this value contented for Saudi Arabia vehicle license plate dimensions. We can see that, the precision rates of our method are very high such as Table 1. In this paper, we have proposed an improved method to segment characters \& numbers in both first row and second row of Saudi Arabia vehicle license plate. In the characters \& numbers recognition module, we have used learning vector quantization artificial neural network to train for characters \& numbers with noises, so the computing time and precision improved. In the using network, we have used the image processing technology for pre-processing to receive high quality of characters \& numbers before putting in the trained networks, so precision rates of the system are higher in the Table 1. In this paper graphical data are shows in Saudi Arabia Vehicle License Plate Segmentation shows in figure 6 and Saudi Arabia Vehicle 


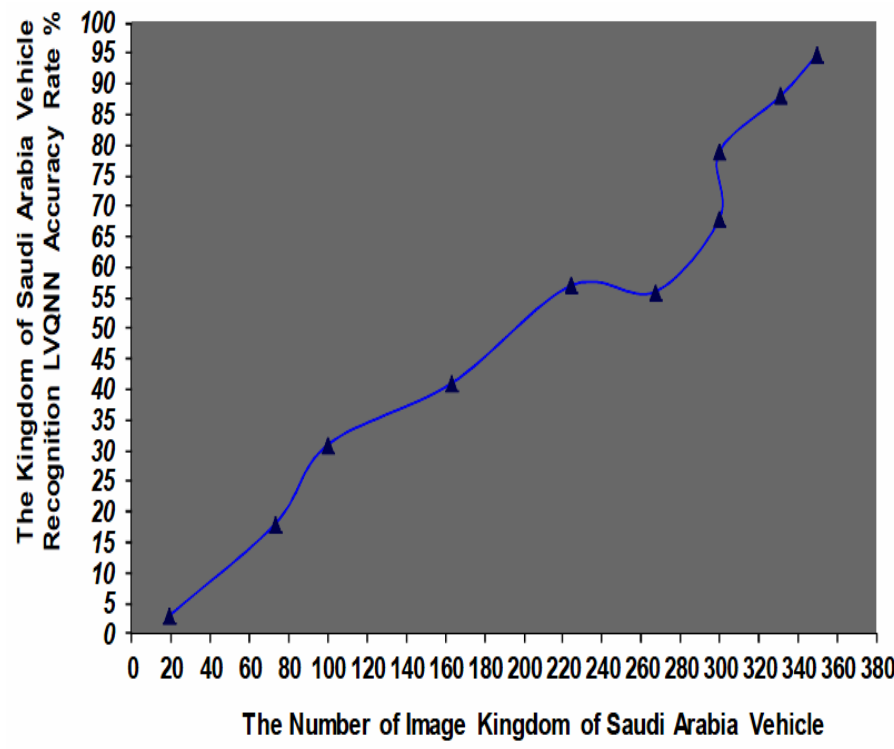

Figure 8. The Overall Kingdom of Saudi Arabia Vehicle License Plate System

Table 1. Results of Character Recognition. Test results of LP Location task, LP Segmentation task, LP Recognition task

\begin{tabular}{|c|c|c|c|}
\hline & $\begin{array}{c}\text { The Saudi Arabia } \\
\text { Vehicle License } \\
\text { Plate } \\
\text { Segmentation }\end{array}$ & $\begin{array}{c}\text { The Saudi Arabia } \\
\text { Vehicle License } \\
\text { Plate Recognition }\end{array}$ & $\begin{array}{c}\text { The Overall } \\
\text { Kingdom of Saudi } \\
\text { Arabia Vehicle } \\
\text { License Plate } \\
\text { System }\end{array}$ \\
\hline The Total Image & 350 & 350 & 350 \\
\hline $\begin{array}{c}\text { The Total Correct } \\
\text { Rate }\end{array}$ & 341 & 337 & 334 \\
\hline $\begin{array}{c}\text { The Total Error } \\
\text { Rate }\end{array}$ & 9 & $91.85 \%$ & $91.6 \%$ \\
\hline $\begin{array}{c}\text { The Arabic } \\
\text { Numerical Rate \% }\end{array}$ & $91.2 \%$ & $96.7 \%$ & $96.55 \%$ \\
\hline $\begin{array}{c}\text { The Arabic } \\
\text { Character Rate \% }\end{array}$ & $96.35 \%$ & $94.91 \%$ & $94.86 \%$ \\
\hline $\begin{array}{c}\text { The Total } \\
\text { Learning Vector } \\
\text { Quantization } \\
\text { Neural Network } \\
\text { Accuracy Rate \% }\end{array}$ & $94.78 \%$ & & \\
\hline
\end{tabular}

License Plate Recognition figure 7 finally the Overall Kingdom of Saudi Arabia Vehicle License Plate System shows in figure 8.

\section{CONCLUSION}

In this paper, we proposed an improved vehicle license plate recognition system for all types of the Kingdom of Saudi Arabia vehicle license plate, which included of characters segmentation and character recognition. In the characters recognition module, we have used a learning vector quantization artificial neural network algorithm to recognize characters \& numbers of the Kingdom of Saudi Arabia vehicle license plate, these networks used independently for Arabic characters \& numbers. The learning vector quantization artificial neural network has trained with noises in the training task. Arabic Character \& number images processed by the pre-processing task, which receive high quality of character \& number images for the using network task to improve precision of the system. It is observed that, as fan beam feature extraction method has more features for training the learning vector quantization artificial neural network thus its simulation precision is higher. Using Fanbeam for feature extraction learning vector quantization artificial neural network is trained. We have tested our improved vehicle license plate recognition system in the Kingdom of Saudi Arabia vehicle license plate images taken from actual system with different conditions like lightening conditions (night \& day), Sandstorm, license angles, illumination, size and type, colors and reflected light. Our vehicle license plate recognition method is more effective than some existing methods earlier, the efficiency of computing time \& accuracy is improved and very satisfied for all types of Kingdom of Saudi Arabia vehicle license plate and Kingdom of Saudi Arabia environment and also more number of Arabic fonts can be used for training the network for improving the precision of the Arabic character recognition.

\section{REFERENCES}

[1] P. Comelli, P. Ferragina,M. N. Granieri, and F. Stabile, "Optical recognition of motor vehicle license plates," IEEE Trans. Veh. Technol., vol. 44, no. 4, pp. 790-799, Nov. 1995.

[2]S.-L. Chang, L.-S. Chen, Y.-C. Chung, and S.-W. Chen, "Automatic license plate recognition," IEEE Trans. Intell. Transp. Syst., vol. 5, no. 1, pp. 42-53, Mar. 2004.

[3] V. Abolghasemi, A. Ahmadyfard, An edge-based coloraided method for license plate detection. Image and Vision Computing 27, pp. 1134-1142, 2009.

[4]Dubey, P., "Heuristic Approach for License Plate Detection", IEEE Conference on Advanced Video and Signal Based Surveillance, 15-16 Sept. 2005, pp. 366370 .

[5]W. Pan, R. An, Morphology and auto -correlation based method of fast locating vehicle license plate. In: Proc. Of the 2nd Internat. Conf. Advanced Computer Control, vol.3, pp. 116-119, 2010.

[6] B.D Ripley, Pattern Recognition and Neural Networks, Cambridge University Press ISBN 052146986 7, 1996.

[7] A. Sato, K. Yamada, Generalized learning vector quantization, Advances in NIPS, MIT Press, pp. 423429, Vol. 7, 1995.

[8]C. Anagnostopoulos, I. Anagnostopoulos, E. Kayafas, and V. Loumos. "A License Plate Recognition System for Intelligent Transportation System Applications", IEEE Trans. Intell. Transp. Syst., 7(3), pp. 377- 392, Sep. 2006.

[9]M. Wu, L. Wei, H. Shih and C. C. Ho. "License Plate Detection Based on 2-Level 2D Haar Wavelet Transform and Edge Density Verification", IEEE International Symposium on Industrial Electronics (ISIE), pp: 1699 1705,2009 
[10]T.-H. Wang, F.-C. Ni, K.-T. Li, and Y.-P. Chen, "Robust license plate recognition based on dynamic projection warping," in Proc. IEEE Int. Conf. Netw., Sens. Control, 2004, pp. 784-788.

[11] Dr. Firoj Parwej, “ A Perceptive Method for Arabic Word Segmentation using Bounding Boxes by Morphological Dilation", for published in the International Journal of Computer Applications (IJCA) USA, Volume 71, No.1, Pages 1 - 7, ISSN 0975 8887, June 2013.

[12] Youngwoo Yoon, Kyu-Dae Ban, Hosub Yoon, and Jaehong Kim, "Blob Extraction based Character Segmentation Method for Automatic License Plate Recognition System", Proceedings of the IEEE International Conference on Systems, Man and Cybernetics, Anchorage, Alaska, USA, IEEE ISBN 9781-4577-0652-3, October 9-12, 2011.

[13] Hossan, M.A. "A Novel Approach for MFCC Feature Extraction", $4^{\text {th }}$ International Conference on Signal Processing and Communication Systems (ICSPCS), pp. 1-5, Dec 2010

[14]P.D. Gader, M. Khabou, Automatic feature generation for handwritten digit recognition. IEEE Trans. Pat- tern Analysis and Machine Intelligence 18(12) 1256\{1262

(1996)

[15] D. Trier , A. K. Jain and T. Taxt, "Feature extraction methods for character recognition-A survey", Pattern Recognition, vol. 29, no. 4, (1996), pp. 641-662.

[16]G. L. Zeng "Nonuniform noise propagation by using the ramp filter in fan-beam computed tomography", IEEE Trans. Med. Imag., vol. 23, no. 6, pp.690 -695 2004.

[17]F. Noo, M. Defrise, R. Clackdoyle, and H. Kudo, "Image reconstruction from fan-beam projections on less than a short scan", Phys. Med. Biol., vol. 47, pp.2525 -2546 2002.

[18] J. W. Beattie, "Tomographic Reconstruction from Fan Beam Geometry Using Radon's Integration Method", IEEE Transactions on Nuclear Science, vol. 22, no. 1, (1975) February, pp. 359-363.

[19] Yusuf Perwej, Firoj Parwej, “A Neuroplasticity (Brain Plasticity) Approach to Use in Artificial Neural Network" for published in the International Journal of Scientific \& Engineering Research (IJSER), France , Vol.3, Issue 6, pp 1- 9, ISSN 2229 - 5518, June 2012.
[20] A. Sato, K. Yamada, Generalized learning vector quantization, Advances in NIPS, MIT Press, pp. 423429, Vol. 7, 1995.

[21] T. Kohonen, The Self-organizing Map, Proceedings of the IEEE, pp. 1464-1480, 1990.

[22] K.L. Wu and M.S Yang, Alternative learning vector quantization, Pattern Recognition Journal, pp. 351-362, Vol. 39, 2006.

[23] M. Biehl, A. Ghosh, B. Hammer, Dynamics and Generalization Ability of LVQ Algorithms, The Journal of Machine Learning Research, pp. 323-360, Vol. 8, 2007.

[24]C. Zhan, X. Lu, M. Hou, and X. Zhou, "A LVQ-based neural network anti-spam email approach", ACM SIGOPS Operating Systems Review Volume 39 , Issue, USA, 2005 pp. 34-39.

\section{AUTHOR'S PROFILE}

Dr. Yusuf Perwej Assistant Professor in the Department of Computer Science \& Engineering Al Baha University, Al Baha , Kingdom of Saudi Arabia (KSA). He has authored a number of different journal and paper. His research interests include Soft Computing, Artificial Neural Network, Machine Learning, Pattern Matching, Pattern Recognition, Artificial Intelligence, Image Processing, Fuzzy Logic, Genetic Algorithm, Robotics, Bluetooth and Network. He is a member of IEEE.

Nikhat Akhtar Assistant Professor in the Department of Computer Science \& Engineering Integral University, Lucknow, India. She has authored a number of different journal and paper. His research interests include Soft Computing, Swarm Intelligence, Storage Technology, Artificial Neural Network, Cryptography, Pattern Matching, Pattern Recognition, Artificial Intelligence, Network Security, Fuzzy Logic, Network and Database. He is a member of IEEE.

Firoj Parwej Assistant Professor in the Department of Computer Science, Jazan University , Jazan , Kingdom of Saudi Arabia (KSA). He has authored a number of different journal and paper. His research interests include Soft Computing, Artificial Neural Network, Machine Learning, Pattern Matching \& Recognition, Artificial Intelligence, Image Processing, Fuzzy Logic, Network and Database. He is a member of IEEE. 